Document downloaded from:

http://hdl.handle.net/10251/59023

This paper must be cited as:

Silvestre-Blanes, J.; Pérez Llorens, R. (2011). Energy efficiency improvements through surveillance applications in industrial buildings. Energy and Buildings. 43:1334-1340. doi:10.1016/j.enbuild.2011.01.017.

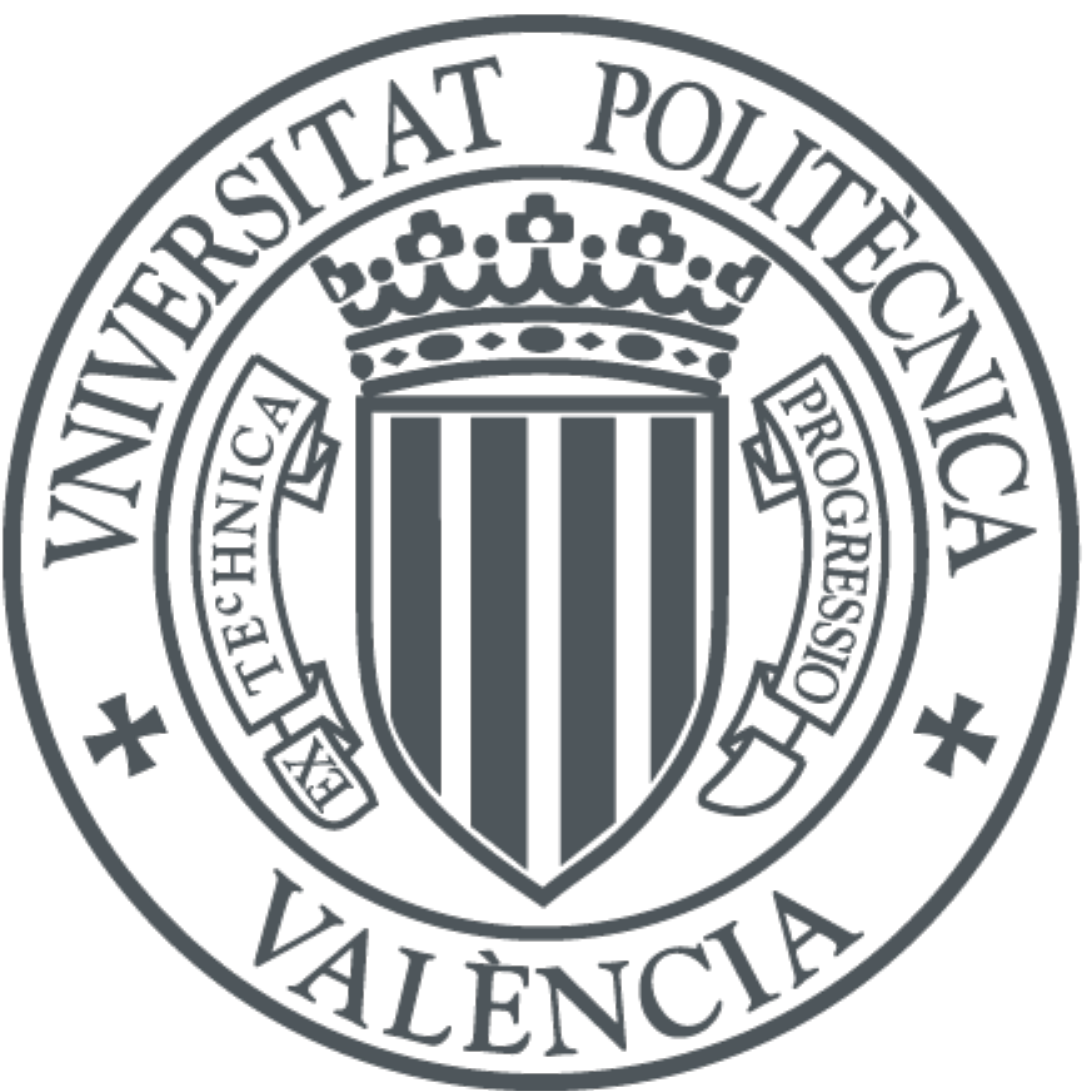

The final publication is available at

Copyright Elsevier

Additional Information 


\title{
Energy efficiency improvements through surveillance applications in industrial
}

\section{buildings}

\author{
Javier Silvestre-Blanes* Rubèn Pèrez-Llorèns \\ Instituto Tecnologógico de Informática (ITI) \\ Universidad Politécnica de Valencia (UPV) \\ Ferrandiz y Carbonell 1. Alcoy 03801. Spain.
}

\begin{abstract}
Presence sensors for energy control based on classic technologies to detect movement are now commonly seen in many areas of life. However, their use in structurally complex environments is not very common, due to their lack of reliability in these types of situations. Falling prices in technologies associated with surveillance applications are leading to a huge increase in their use in all types of environment, with monitoring of traffic and people the most common of these. In this work, we carry out an analysis of occupancy patterns in manufacturing industries with the aim of determining the possible energy savings that could be obtained using these new technologies. We also carry out an analysis of the possibilities of using these technologies as presence sensors, analyzing the trends and limitations associated with them.
\end{abstract}

Key words: Intelligent building, Occupancy detection, Energy efficiency, Image processing 


\section{Introduction}

Outside the area of security, presence sensor technology has, since its introduction, also been widely used as a tool for more efficient energy consumption control. Institutions such as the Electric Power Research Institute (EPRI) and the American Society of Heating, Refrigerating, and Air-Conditioning Engineers (ASHRAE) estimate an average rate of saving of $30 \%$ through the use of presence sensor technology in commercial buildings [1][2]. Other studies rate the possible savings at $25-50 \%$ in offices containing two people [3], while further studies in different countries and situations have sugested possible reductions of between $35 \%$ and $75 \%$ [4][5]. These technologies are based on passive infrared (PIR) sensors or on ultrasonic technology [6] which, in spite of the improvement they provide (generally considered to average out at around 30\% [7]) have significant limitations regarding the optimization of energy efficiency. In addition, inefficient use and poor installation of this kind of system can in fact increase energy consumption instead of reducing it [8]. For this reason, the installation and functioning of energy efficiency systems must be kept simple, and should be of the plug and play type, not requiring complex parameter setting operations. Other more recent studies [7] have tried to overcome these shortcomings by using probabilistic models (belief networks) applied to redundant presence sensors, which make it possible, to a certain degree, to improve efficiency compared to the use of conventional presence sensors. However, these studies have fallen far short of the maximum level of achievable energy efficiency. In [9] a study was carried out which showed a

\footnotetext{
* Corresponding Author

Email address: jsilves@disca.upv.es. tel.:+349966528545 (Javier

Silvestre-Blanes).
} 
significant correlation between number of occupants and certain environmental measurements which can give $75 \%$ accuracy in number detection. These results were obtained for office environments and so it is difficult to apply these results to industrial environments where the parameters measured may have no relationship with number of occupants (for example, acoustic measurements).

One of the problems with current technology is the coverage of motion detectors, as they usually have much lower levels of coverage when compared to multimedia technology (7.6 meters of distance [10]), which hinders the installation and configuration of energy saving systems. Moreover, it is necessary that movement takes place in the scene, which creates important restrictions:

- firstly, it is possible that there is human presence but due to their activity, a low-level motion activity, the sensor does not detect movement so the lights are wrongly turned off after some time with no apparent activity, causing serious problems. These problems can create risk situations, especially in industrial application environments.

- secondly, and especially in industrial environments, movements are often detected which have been caused by machines and robots. These systems, which are unable to distinguish between human and machine movement, would constantly turn on the lights unnecessarily, which would cause very low energy efficiency, far below the estimated values in the studies mentioned previously of between $25 \%$ and $75 \%$.

In order to deal with this first problem, a delay in turning off the light is configured (TD: Time Delay), which is normally a constant value, usually recommended to be between 5 and 30 minutes [10], depending on the type of room. This can mean low energy efficiency in most industrial environments, 
even when setting this parameter to relatively low values, which is not always possible, as we mentioned before, due to safety reasons. It is not possible to overcome the second problem with this technology, which requires very careful installation in order to achieve energy efficiency.

To improve energy optimisation, some studies have determined that it is necessary to know the occupancy patterns of a particular situation to improve the attainable savings [11]. However, most of these studies are now a little out of date and they have not comprehensively analyzed the possible savings in complex industrial environments. In this study the occupancy model of modern industries are analyzed, including some complex environments where classic presence sensors cannot be applied. The occupancy models are obtained using multimedia technology. In the next section, there is a review of the multimedia technology. In section 3 the industrial occupancy models are presented. Next, some cases are analyzed from the point of view of their technical and economical viability using multimedia technology. Finally, conclusions and future work are presented.

\section{Multimedia Energy Control System}

To develop the ocupancy models and set up a presence control system based on multimedia technology, it is necessary to install, in each scenario, a set of equipment that allows capture of images with the necessary velocity and resolution to allow an image processing algorithm to process it and detect human presence, the movement of their activity, and also which can interface with the lighting control system, reducing consumption in cases where there is no human activity (see Fig. 1). These systems also consume power so, is it 
possible to save energy by using multimedia technology?

\subsection{Multimedia applied to this field}

An in-depth analysis of image processing and multimedia technology is outside of the scope of this paper, however, a brief review is necessary. The multimedia technology that is applicable in this field is based on that developed for surveillance systems (see Fig. 2). These are based in the following software blocks (see [12]): a separation between the background (BG) and the foreground (FG); blob detection in the foreground image; blob classification; blob tracking and analysis algorithm. The computing cost for the BG/FG separation may be high, but is fundamental for the correct execution of the following stages. The most common techniques are background supression, background subtraction, and variations on the two [13]. Other more developed techniques are based on Kalman filters or Mixture of Gaussians, see [14], to determine the colour distribution of each pixel belonging to a stable BG object. Object detection techniques have lower complexity, as they are based on calculation of the connected components of the mask obtained in the previous module. It also analyzes the difference in position with respect to previous frames and updates the path of objects of interest where necessary. Often, the objects of interest are people or vehicles, and there are many techniques for the detection of these elements [15][16][17][18][19]. 


\subsection{Problem formulation}

To answer the previous question, we need to know the relationship between the energy that can be saved, and the energy needed by the multimedia system. If $e_{L}$ is the energy consumed by each light without using the lighting control system and in a particular scenario there are $m$ lights, the global light energy needed by the scenario is $E_{L}=m e_{L}$. Then, the energy consumption of the control system must be significantly lower than the attainable saving. The control system has to take into account the energy consumed by cameras Es, the energy used in the Ep image processing node and the energy used by the lighting control system Ec. Through the use of this multimedia control system the energy needed by the lights is reduced to $E_{l}$.

Then, the energy used by control system must be lower that the energy saved, So:

$$
E_{s}+E_{p}+E_{c}<<<E_{L}-E_{l}
$$

In this way, it will be possible to obtain a reduction coefficient $C_{r}=1-\left(E_{s}+\right.$ $\left.E_{p}+E_{c}+E_{l}\right) / E_{L}$ which allows us to obtain the savings ratio that it is possible to achieve.

On the other hand, it is necessary to analyze which parameters influence the possible achievable savings. To carry out this analysis, we denominate the energy used by one light as $e_{s}$ using the save power mechanism while $e_{l}$ and $e_{L}$ are the lower and higher energies used by the light. If $r^{i}$ is the ratio of time where the light $i$ must be at maximum energy rate due to the application 
requirements, the light energy used by one light $i$ can be defined as:

$$
e_{s}^{i}=r^{i} e_{L}^{i}+\left(1-r^{i}\right) e_{l}^{i}
$$

For simplicity, we assume that the mean of $r^{i}$ is the same for all lights covered by the sensors in one scenario, and they are also always of the same kind, so we can calculate $E_{l}$ as:

$$
E_{l}=\sum_{i=1}^{m}\left(r e_{L}+(1-r) e_{l}\right)
$$

Concerning $E s$, this will depend on the number of sensors $n$ and on the energy used by one sensor $e_{s}$ including acquisition and transmission (and encoding if neccesary). This will depend on the frequency $f$ of the acquisition, so $E_{s}=$ $n e_{s}(f)$. We have to consider that the energy consumed by the processing node will depend on the power energy needed by the algorithm to process a stream with frequency $f$, that is $e_{p}(f)$, and that the processing node deals with $n$ sensors. Thus $E_{p}=n e_{p}(f)$. On the other hand, if $e_{c}$ is the energy to manage one light, then $E_{c}=m e_{c}$. Equation (1) can be expressed as:

$$
e_{s}(f)+e_{p}(f)<\frac{m}{n}\left((1-r)\left(e_{L}-e_{l}\right)-e_{c}\right)
$$

This equation shows that the key parameters to maximize energy saving are $r$, the achievable difference in consumption per light $\left(e_{L}-e_{l}\right)$ and the relationship $m / n$ between the number of lights and the number of sensors necessary for their control. These parameters are dependent on the application and its environment, and therefore there are constants which limit the achievable power saving. Other variables which influence this are $f$, and the influence of this value on $e_{s}$ and $e_{p}$. Moreover, $f$ as well as $n$ also influence the reliability of the system, limiting the maximum power saving achievable. 


\section{Industrial Ocupancy Models}

For the development of the occupancy models that allow us to obtain estimates of $r$, different scenarios representing industrial situations were identified. Some that were highly automated and complex were selected, as well as others which, although automated, involved more movement and occupancy by human operators. It is important to take into account that the value of $r$ itself is not enough, it is also necessary to know the occupancy pattern in order to be able to evaluate the possible achievable savings. If the occupancy pattern is favorable (e.g. $50 \%$ with 12 hours on and 12 off) real savings are possible but in the opposite case (e.g. $50 \%$ with 20 seconds on and 20 seconds off) savings are not realistically possible. In table 1 we can see the scenarios analyzed in the occupancy models, and the annual consumption $E_{L}$ in KWh, taking into account the light systems used and the hours per day when they are turned on during the year depending on the characteristics of each scenario. Images of these scenarios are shown in Fig. 3, where we can also see the unmasked area of the images where motion analysis of the scenes was carried out. Scenarios 1 and 2 correspond to the same industrial production room. However, scenario 1, the corridor, is a production area of much activity, while scenario 2 is a passage and temporary storage area. The length of both corridors is the same, using 15 fluorescent lighting fixtures of $2 \times 58 \mathrm{~W}, 12$ months a year, 7 days a week and 24 hours a day. Scenario 3 covers the presence control system and it is also a passage area. In this case, 3 fluorescent lighting fixtures of $2 \times 58 \mathrm{~W}$ are used, but only for 5 days per week and 11 months per year. In the next scenario the room is occupied by a production machine that requires a lot of attention by the operator. 4 fluorescent lighting fixtures of $2 \times 58 \mathrm{~W}$ are 
used 5 days per week during 11 months of the year. Scenario 5 is a storage area with 16 fluorescent lighting fixtures of $2 \times 58 \mathrm{~W}$. It is considered to be in use 5 days per week, 11 months per year. In scenario 6 , access to a totally automated packing machine is controlled. Human intervention is not required except for very precise cases. However, 9 fluorescent lighting fixtures of $2 \times 58 \mathrm{~W}$ cover this area, permanently turned on 11 months of the year. Scenario 7 is a highly automated area also, although this area requires attention by the operators at the beginning and the end of each process (processes can last from 8 to 36 hours, depending on the characteristics of the production in question). Scenarios 8-11 are the most complex of all scenarios analyzed in this paper. Fig. 4 shows a diagram of the area that is controlled. The cameras have been placed at the two access corridors that connect the working areas. The high level of automation of the machinery means that this section is controlled by a single operator who is also doing other tasks, so he does not spend $100 \%$ of his time in this section. In each working corridor there are 16 fluorescent lighting fixtures of $2 \times 58 \mathrm{~W}$, while the access corridors are covered by 5 fluorescent lighting fixtures of $2 \times 58 \mathrm{~W}, 24$ hours per day, 11 months per year.

To develop the occupancy models, we obtained histograms (see Fig. 5) which show the frequency of period between two instants of time where human activity required lighting. Axis $X$ shows the units of time in minutes up to 60 minutes, and then in groups of one hour. This information makes it possible to analyse the periods of time where the frequency of the lighting use could have been reduced with the resulting energy savings.

As we can see in the histogram for scenario 1, in this case the level of human activity is very high which limits to a great degree the possibilities of energy saving in lighting, since the periods of significant human activity are few and 
short. Considering only the periods of inactivity longer than 10 minutes, the saving would be $1 \%$. By reducing this value to 5 minutes, it would only reach $4 \%$. Therefore, in this kind of industrial scenario, the use of devices for energy saving are neither viable nor profitable. In scenario 2 , in the same industrial plant as scenario 1, as it is a passing and temporary storage area, and considering it viable to reduce the lightning to 5 minutes of inactivity, an estimated reduction of $50 \%$ would be achieved, reducing the consumption to $7,700 \mathrm{KWh}$. In scenario 3, the information is captured at the operator presence control point. As we can see in the graph, the periods of inactivity are very high, and would be higher if it were not a common passage area. By considering the reduction of lighting in periods of inactivity longer than 10 minutes, the consumption could be reduced to $5 \%$ of the current consumption, reducing it to $10 \mathrm{KWh}$. In scenario 4, we analyze the activity observed at a packing machine that requires the intervention of an operator. As we can see in the histogram, periods of high activity are alternated with other periods where human activity is lower. This can be caused by the periods of inactivity of the production machine or by any other circumstance. In any case, the situation is representative of a great number of situations. In this case, the consumption of 2,449.920 KWh could be reduced by $50 \%$ considering that the lights have been turned off in every period of inactivity longer than 10 minutes, reducing the consumption to 1,230 KWh. In scenario 5, we can see a great variation in levels of human activity. This is normal and logical in the case of a warehouse, where there are long periods of inactivity and other periods where different operators enter to remove or store a certain material. Considering the periods of inactivity longer than 10 minutes, the consumption could be reduced from 9,799.680 KWh to about 2,000 KWh, resulting in a very significant saving. Scenario 6 is a typical example, representative of a highly automated indus- 
trial activity. In this kind of scenario there are significant periods of human inactivity occurring frequently. It shows a very low occupancy pattern, with very long periods of inactivity. Considering only the periods of inactivity lower than 50 minutes, it would mean that for $50 \%$ of the time there is no activity at all. This could mean a saving of approximately $50 \%$ of the $8,264.900 \mathrm{KWh}$ this section shows. Considering periods of inactivity longer than 10 minutes, the saving would be of $95 \%$, that is, the consumption could be reduced from 8,393.760 KWh to $420 \mathrm{KWh}$, approximately. In scenario 7, although there are very long periods of human inactivity, this is not as frequent as in the previous case. Considering only the periods of inactivity longer than 10 minutes, this means approximately $60 \%$ of the total time. Therefore, an energy control system in this scenario could reduce the consumption from 9,187.200 KWh to 3,800 KWh.

Scenarios 8-11 (see Fig. 4) do not provide enough information to carry out an analysis with the same precision as those previously described. This is because in these cases a 3D calibration of the scenarios would be necessary along with an increase in capture frequency and higher resolution in the sensors (or in certain cases a higher number of sensors). Analyzing the data available, and considering the periods of human inactivity longer than 5 minutes, for each of these, savings of $4.3 \%, 16.1 \%, 42 \%, 21 \%$ would be obtained. This data makes sense and reflects the reality of scenario 8 which, as it is a passage area, shows more activity than the other areas. A pessimistic estimate of the achievable saving in energy would be about $70 \%$, which would lower the consumption by more than 50,000 KWh. 


\section{Case Study}

In order to analyse the viability of multimedia control, we have analyzed the use of this technology in some of the scenarios described here, in particular, those where energy savings are considered possible with the right devices, and it is difficult or impossible to achieve these savings via conventional methods. Fig. 6 shows the savings achievable depending on $C_{r}$ and on the $E_{L}$ of the scenario, assuming $0,15 € / \mathrm{KWh}$. These savings have to be higher than the cost of the equipment. Thus, scenarios 2,6 and 8-11 were selected for the analysis. The multimedia equipment is the same as that used in the compilation of the occupancy models. The cameras used were Panasonic BL-C1, with a maximum resolution of 640x480, which transfer the image compressed in JPEG to a processing node through Ethernet, at 3 images per second $(\mathrm{f}=1 / 3)$, with a consumption of $1.7 \mathrm{~W}$. For the processing, we used a laptop with a Pentium M processor with a scaling of 800, 1067, 1333 and $1733 \mathrm{MHz}$ frequencies, and the consumptions shown in table 2 depending on the algorithm used and the value of $f$. The worst case scenario is considered, that is, $e_{p}=31 \mathrm{~W}$ and the algorithm with best performance in the detection and tracking which saturate the processing node at $\mathrm{f}=2$. One processing node is used for each sensor in the analysis performed. Concerning the lighting, through the use of dimmable ballast $1-10 \mathrm{v}$ we obtained a $e_{L}=55 \mathrm{w}$ and $e_{l}=10 \mathrm{w}$. For a cost analysis, we can consider $100 €$ for the sensor, $400 €$ for the processing node, and $500 €$ for a light control system based on DALI [20] (Digital Addresable Lighting Interface). It is assumed that the cost of DALI ballast is not considered, since the differences in prices with conventional electronic ballast is negligible 
With this data, in scenario 2 , where $m=15$ and $n=1$, it is possible to obtain $C_{r}=0.37$. This would allow a energy consumption reduction of $5,950 \mathrm{KWh}$, and savings of $892 €$ per year, which is enough to make the necessary investment in the system profitable. In the case of scenario 6 , for which $m=9$ and $n=1$, we can achieve $C_{r}=0.72$. Although the saving ratio is much higher than in the previous scenario, the original consumption is also lower, meaning that the difference is not excessive, reaching a saving of 5,870 KWh, and savings of $893 €$ per year, meaning that again the intial set up cost of the system would be quickly repaid. In the case of scenarios 8-11, using $n=4$ and a processing node for each sensor, and under the conditions described in the previous section, $C_{r}=0.55$ could be achieved, which would represent

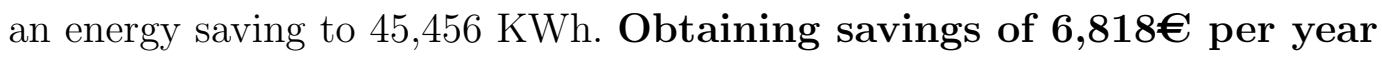
would compensate for the increase of $n$. However, as has been highlighted in the previous section, the use of $n=4$ does not provide enough information to control the lighting system. A more realistic approach would be to use a camera/processing node on each side of each working corridor. Then, using $n=10$, the $C_{r}$ is reduced to 0.51 , which represents a reduction in energy consumption to $42,150 \mathrm{KWh}$. the increase of $n$ produces savings of $6,322 €$ per year. This reduction compensates for the increase in the number of sensors which are needed to guarantee correct working of the system without significantly reducing the value of $C_{r}$. 


\section{Conclusions and future work}

The use of conventional presence sensors in industrial environments, especially in situations which are complicated by spatial distribution, automated systems, characteristics of the human activity involved, etc., is not always feasible. However, in these environments, we often see inefficient use of energy, as there are no mechanisms that guarantee lighting only when there is human presence, allowing a saving in other cases. Therefore, depending on safety regulations of the country involved (which may mean that lights must be permanently on, when in reality the need for them is very low) the use of surveillance technologies to reduce power consumption can give very significant results.

The parameters which determine the achievable energy savings and the technological viability of the system are: firstly, the occupancy pattern, which is the first element to analyze as it tells us if we should discard the possibility of energy savings whichever technique is used; secondly, the relationship between the number of lights $m$ and the number of sensors/processing nodes necessary $n$, as well as the difference in consumption between maximum and minimum power $\left(e_{L}-e_{l}\right)$, which also determine possible energy savings. Power consumption $e_{p}$ is only relevant if the ratio $m / n$ is quite low. However, the analysis of surveillance algorithms is of great interest in any case independant of the $m$ and $n$ of the application. Firstly, this reduction allows us to increase the frequency without an increase in number of processing nodes to obtain better results in the application. Secondly, it also allows us to increase the number of areas covered by the same processing node. Finally, given the recent explosion in surveillance applications, the reduction in consumption in sensors as well 
as in image transmission and processing nodes presents both ecological and economic advantages.

We are currently working on vision modules to obtain good performance in segmentation and tracking of the object as the objective of the system. The algorithm has to take into account the lower illumination conditions in the first blob detection, and the frequency considerations according to the blob velocity, sensor distribution, image frequency and energy considerations.

\section{Acknowledgements}

\section{References}

[1] L. Audin, Occupancy sensors: promise and pitfalls, E-Source Tech Update, Old Snowmass, CO, 1993.

[2] EPRI, Occupancy sensors: positive on/off lighting control, EPRI-BR-100323, Palo Alto, CA, 1994.3.

[3] California Energy Commission (CEC), Advanced lighting guideliness, CEC Publication 400-93-014, Sacramento, California Energy Commission, 1993.

[4] M.S. Rea, R.R.Jaekel, Monitoring occupancy and light operation, Lighting Research and Technology 19 (1987) 45-49.

[5] DOE, Advanced lighting guideliness, US Departament of Energy, 1993.

[6] Vishal Garg, N.K. Bansal, Smart occupancy sensors to reduce energy consumption, Energy and Buildings 32 (2000) 81-87.

[7] Robert H. Dodier, Gregor P. Henze, Dale K. Tiller, Xin Guo, Building 
occupancy detection through sensor belief networks, Energy and Buildings 38 (2006) 1033-1043.

[8] D.B. Floyd, D.S. Parker, J. R. Shervin, Measured field performance and energy savings of occupancy sensors: three case studies, Florida Solar Energy Center, Cocoa, http://www.fsec.ucf.edu/ bdac/pubs/PF309/PF309.html.

[9] Bing Dong, Burton Andrews, Khee Poh Lam, Michael Höynck, Rui Zhang, An information technology enabled sustainability test-bed (ITEST) for occupancy detection through an environmental sensing network, Energy and Buildings 42 (2010) 1038-1046.

[10] Legrand, Quick guide to reducing lighting energy use at home. Watt stopper,

[11] D. Manniccia, Predicting wasted lighting energy through occupancy monitoring, In: Proceedings of the IESNA, 1993.

[12] Trista P. Chen, Horst Haussecker, Computer Vision Workload Analysis: Case Study of Video Surveillance Systems, Intel Technology Journal 9 (2005) 109118.

[13] I. Haritaoglu, D. Harwood, L. David, Real-Time surveillance of people and their activities, IEEE Trans. Pattern Analysis and Machine Intelligence 22 (8) (2000) 809-830.

[14] L. Li, W. Huang, Irene Gu, Q. Tian, Foreground object detection from videos containing complex background, In: Proceedings of the 11th ACM international conference on Multimedia, November 2-8, Berkeley, CA, USA, 2003.

[15] P. Viola, M.J. Jones, D. Show, Detecting pedestrians using patterns of motion and appareance, In: Proceedings of Int. Conf. in Computer Vision, Octuber 13-15, Nice, France, 2003.

[16] H. Scheiderman, T. Kanade, A statistical model for 3D object detection applied 
to faces and cars, In: Proceedings of Int. Conf. in Computer Vision and Pattern Recognition, June 13-15, Hilton Head, SC, USA, 2000.

[17] F. Porikli, O. Tuzel, Human body tracking by adaptative background models and mean-shift analysis, Mitsububhi Electric Research Laboratory, TR-200336, 2003.

[18] X. Desurmont and A. Bastide and C. Chaudy and C. Parisot and J.F. Delaigle and B. Macq Image analysis architectures and techniques for intelligent video surveillance systems IEE Proceedings, 2005, 224-231

[19] Michal Kawulok Energy-based blob analysis for improving precision of skin segmentation Multimedia Tools App (2010) 49, 463-481

[20] Francis Rubinstein and Stephen Treado and Peter Pettler Standarizing communication between lighting control devices Industry Applications Conference (2003) 805-811 


\section{List of Figures}

1 Generic Scenario 19

2 Surveillace System Blocks 19

$3 \quad$ Images of scenarios 20

$4 \quad$ Example of application. Scenarios 8-11. 21

$5 \quad$ Ocupancy models 22

$\begin{array}{lll}6 & \text { Savings achievable } & 23\end{array}$ 


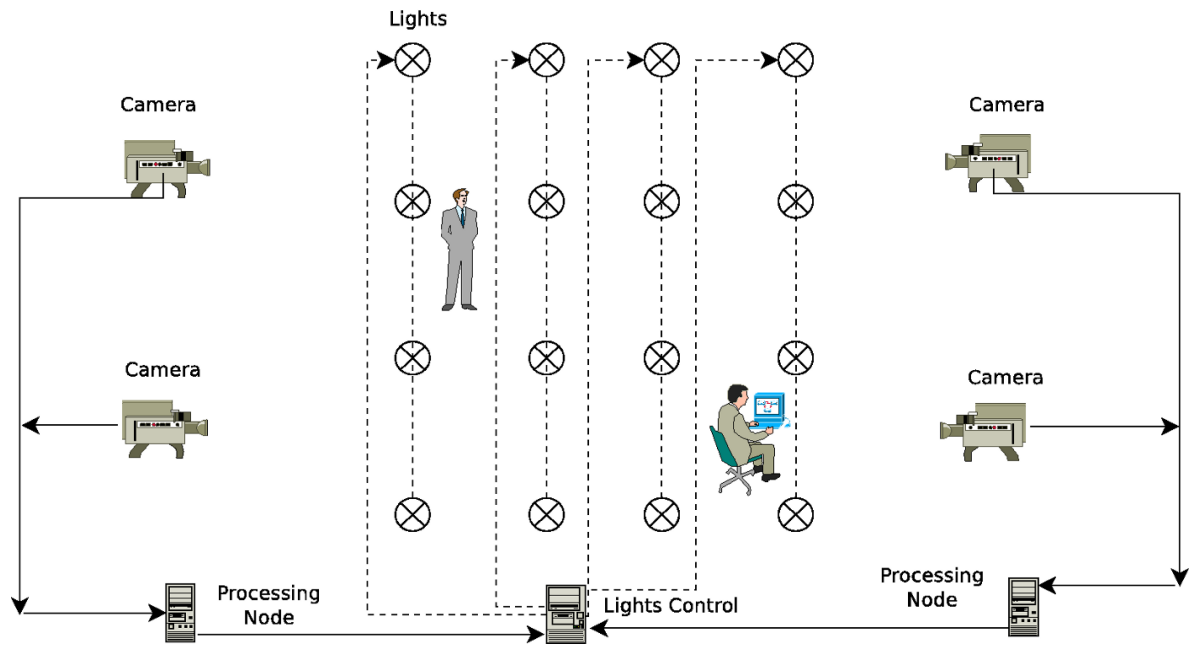

Fig. 1. Generic Scenario

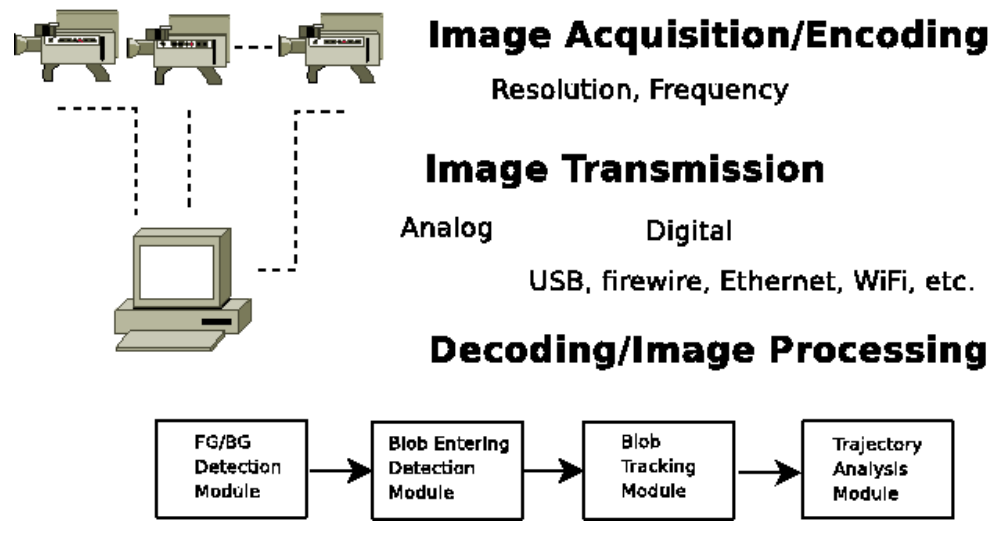

Fig. 2. Surveillace System Blocks 


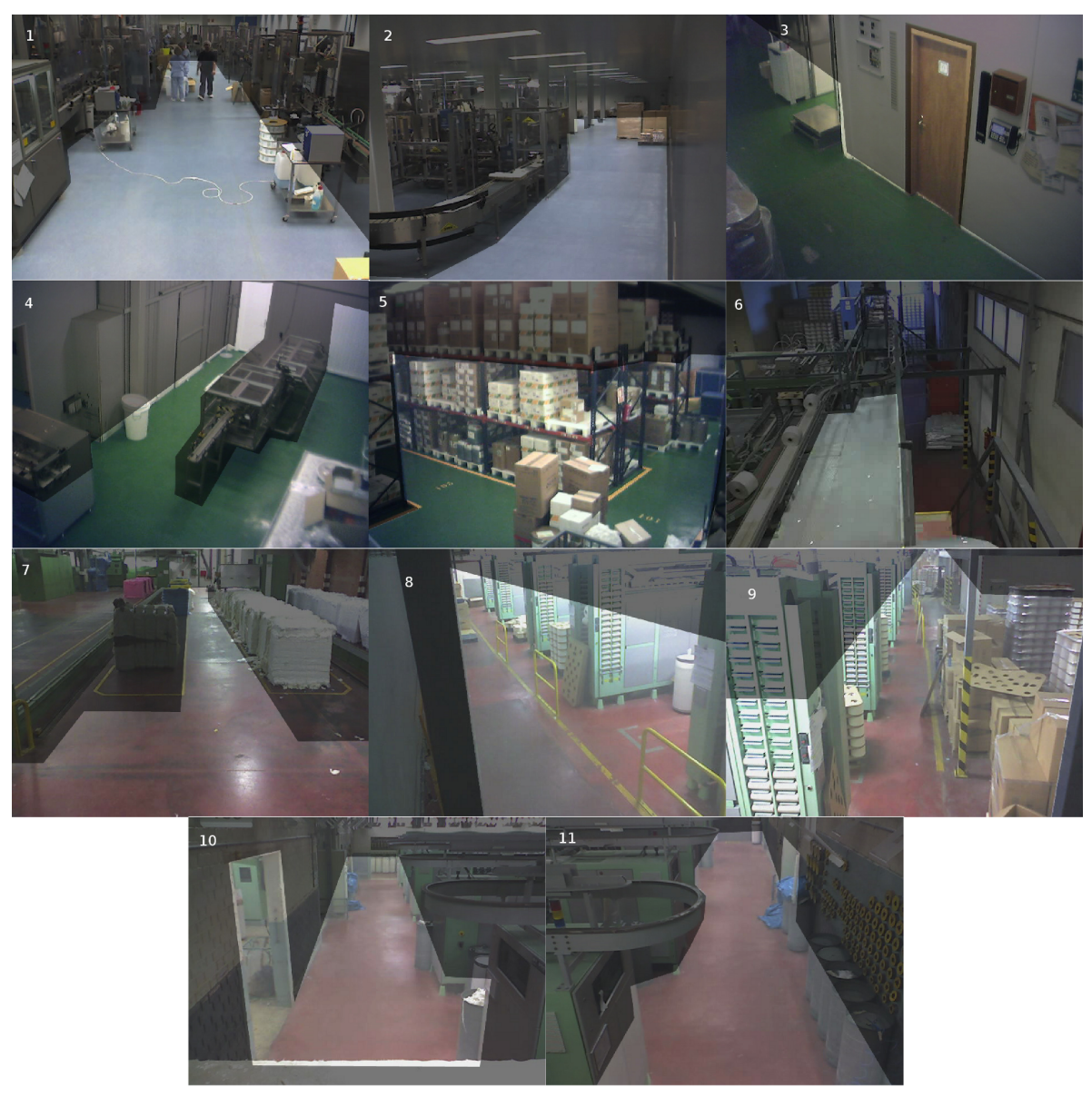

Fig. 3. Images of scenarios 


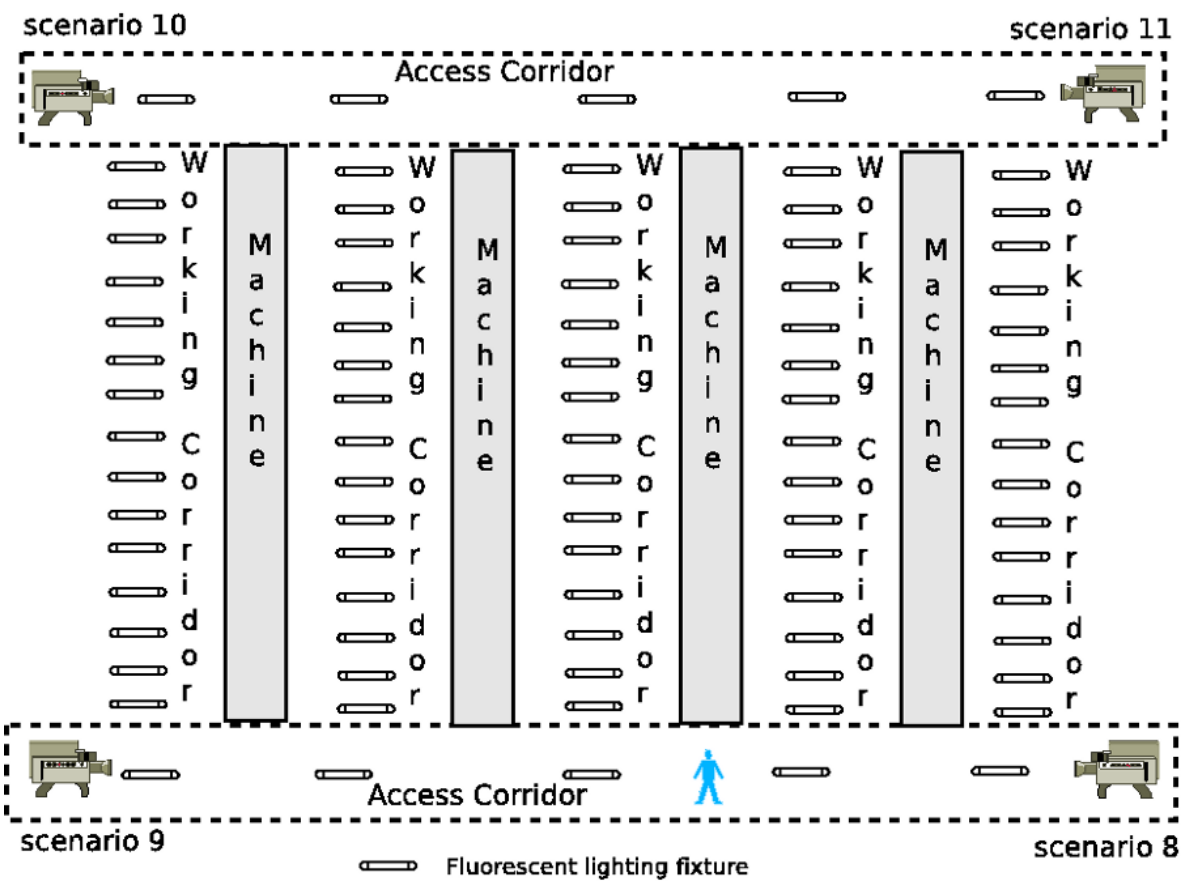

Fig. 4. Example of application. Scenarios 8-11. 

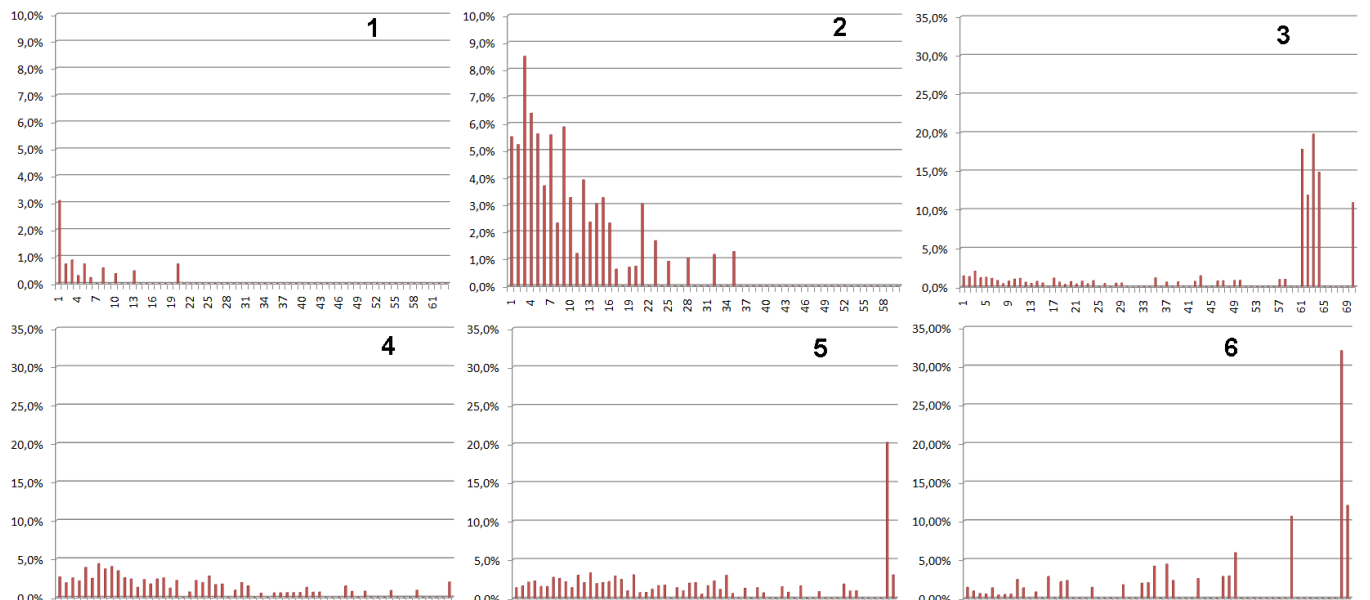

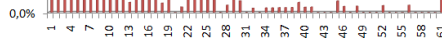

0\%

|| || || || || || || ||
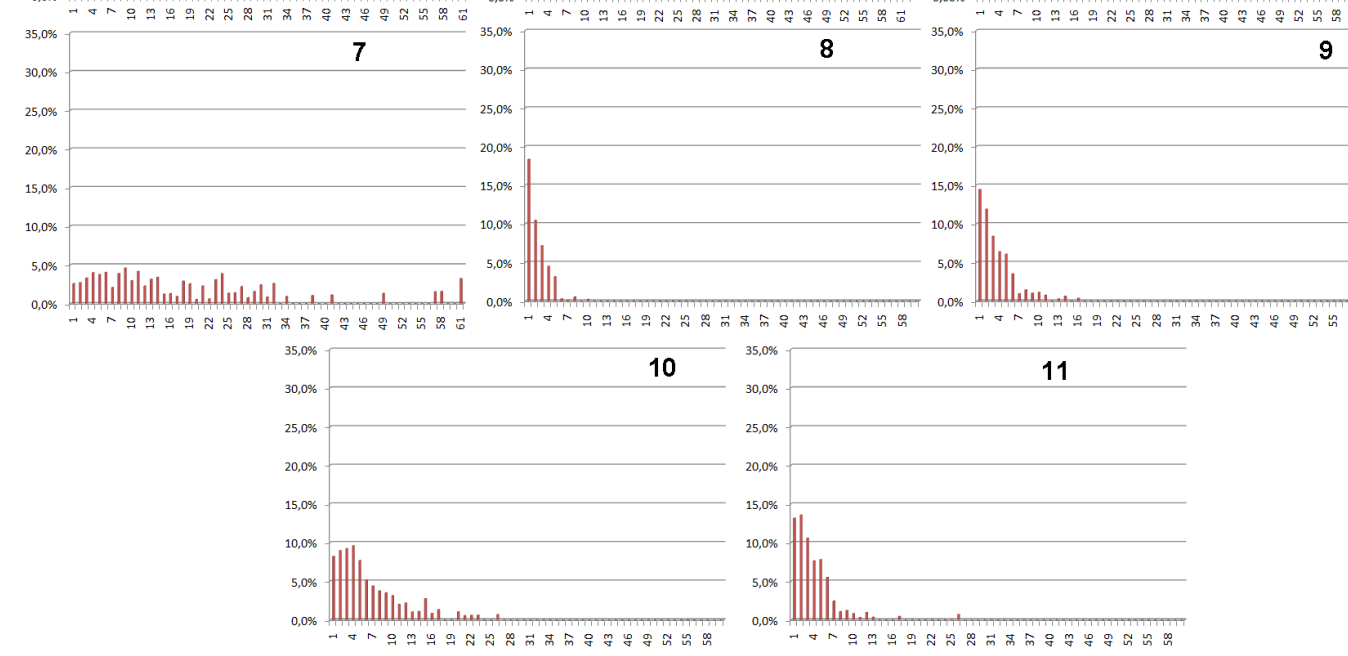

Fig. 5. Ocupancy models 


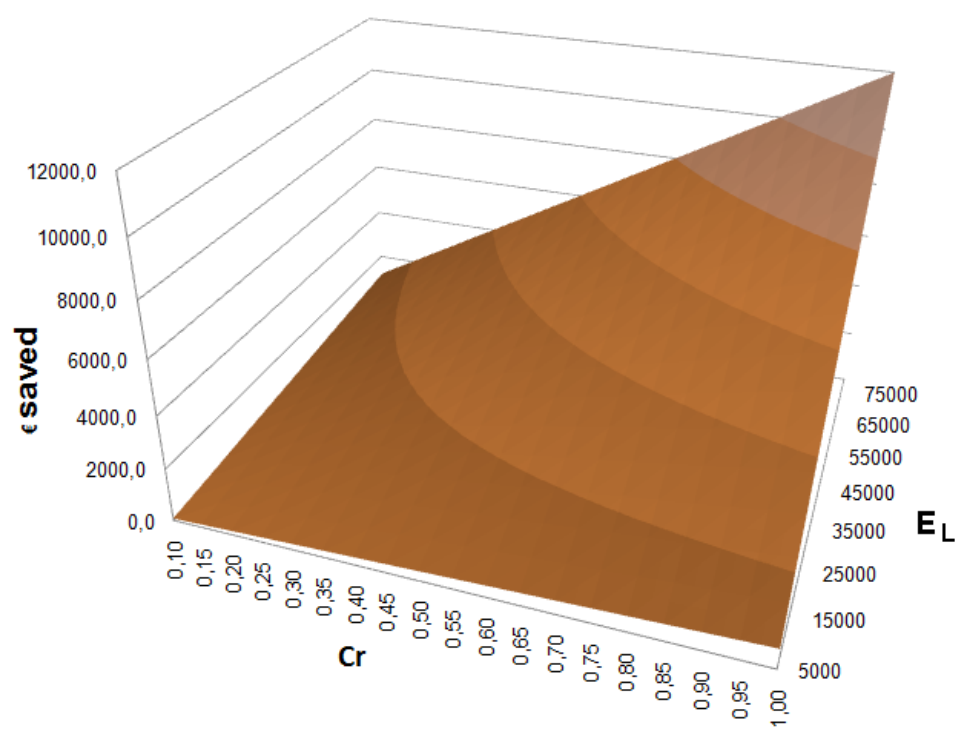

Fig. 6. Savings achievable 
Table 1

Power consumption scenarios

\begin{tabular}{|c|c|c|}
\hline scenario no. & place & $E_{L}(\mathrm{KWh} /$ year $)$ \\
\hline 1 & Pharmaceutical plant. Working corridor & $15,242.40$ \\
\hline 2 & Pharmaceutical plant. Access corridor & $15,242.40$ \\
\hline 3 & Present control and access corrodor & $1,837.44$ \\
\hline 4 & Production machine & $2,449.92$ \\
\hline 5 & Warehouse & $9,799.68$ \\
\hline 6 & Automatic packing & $8,264.9$ \\
\hline 7 & Preprocess & $9,187.20$ \\
\hline $8-11$ & Textil spinning & $82,684.80$ \\
\hline
\end{tabular}


Table 2

Power consumption

FG/BG Blob Detection Blob Tracking power at 1fps power at $4 \mathrm{fps}$

\begin{tabular}{l|ccccc}
\hline Alg. 1 & FG_0s & BD_simple & CC & $16 \mathrm{~W}$ & $31 \mathrm{~W}$ \\
Alg. 2 & FG_0 & BD_simple & CC & $18 \mathrm{~W}$ & $31 \mathrm{~W}$ \\
Alg. 3 & FG_1 & BD_CC & MSPF & 21W & $31 \mathrm{~W}$ \\
Different configurations of blobtrack tool (see tool documentation)
\end{tabular}

